\title{
Robust License Plate Detection using Convolutional Neural Network
}

\author{
I Nyoman Gede Arya Astawa ${ }^{1}$, I Gusti Ngurah Bagus Caturbawa ${ }^{2}$, Elina Rudiastari ${ }^{3}$, I Made Ari Dwi Suta Atmaja ${ }^{4}$ \\ Department of Electrical Engineering \\ Politeknik Negeri Bali \\ Bali, Indonesia \\ 1 arya_kmg@pnb.ac.id, ${ }^{2}$ caturbawa@pnb.ac.id, ${ }^{3}$ lina_rudiastari@pnb.ac.id, ${ }^{4}$ arisuta@pnb.ac.id
}

\begin{abstract}
In general, the vehicle number plate detection system in an image should be able to overcome two problems, first how to determine the position of the vehicle number plate or which vehicle number plate is on an image and the second how big is the plate. A number of vehicle license plate detection methods have been proposed over the past two decades, and some have shown success in certain tasks. This study will detect the license plate number of vehicles using the CNN. The initial process is to create a training data license plate numbers using CNN processed on the server. Furthermore, the training data entered on the vehicle license plate detection applications. Vehicle license plate detection results will be displayed in percent accuracy. Based on the testing, the results obtained were very satisfactory with the accuracy of detection of vehicle license numbers as much as $98.19 \%$, the remaining $1.81 \%$ of the plates were detected but not the image of the plate that was cropped. It shows that the method has been implemented on CNN that training data and license plate detection is very pretty accurate in detecting vehicle license plate. Implementation of $\mathrm{CNN}$ methods on android based mobile devices for data processing training and detection testing of vehicle license plate shows very satisfactory results.
\end{abstract}

\section{Keywords - license plate, detection, CNN, mobile device}

\section{INTRODUCTION}

Mobile phone devices are not just limited to communication devices. By using the application of computing technology, a mobile phone device can perform different functions even at the same time. Implementation of the operating system in mobile devices make the device capable of computing activities that are almost the same as computing devices in general. This makes mobile devices possible to add applications based on artificial intelligence (AI) such as face recognition, fingerprint recognition, vehicle license recognition, etc.

License plates detection has a role in smart transportation systems. This can be applied in many applications such as speed measurement, vehicle recognition, estimated traffic flow, security controls, automatic vehicle tickets, traffic monitoring etc $(1,2)$. There are several journals that have reported the results of license plate detection studies with very good results, even with almost perfect accuracy in their test datasets, but vehicle number plate detection in an open environment is still a challenging research (2). There are some difficulties in detecting license plates such as non-uniform backgrounds, blur, inappropriate camera settings, shadows, and illuminations. These difficulties will often be found when a license plate detection is implemented on mobile phone devices.

In general, the vehicle number plate detection system in an image should be able to overcome two problems, first how to determine the position of the vehicle number plate or which vehicle number plate is on an image and the second how big is the plate. A number of vehicle license plate detection methods have been proposed over the past two decades, and some have shown success in certain tasks (3). The researcher (2) uses the principal visual word (PVW) method by determining the position of the candidate character in the number plate identified for the first time, and the bounding box of the plate is determined later and the result can automatically detect the vehicle number plate. The researcher (1) models the function that produces scores for each image sub-region using the convolutional neural network (CNN) method which allows to estimate the number plate location detected by several vehicles on several highway lanes. $\mathrm{CNN}$ and Recurrent Neural Network (RNN) methods are used by researchers (4), both methods have advantages in learning features and joint image or embedding labels. The results obtained are the performance of these methods comparable to manual engineering methods such as the sliding window method. The license plate detection study conducted by (5) based edge-geometrical features using morphological approach. Researchers (3) used the line density filter (LDF) method to extract candidate plate areas, thereby significantly reducing the area to be analyzed for plate localization. Researchers (6) using sliding window method, histogram of oriented gradients and support vector machines for detection of vehicle license plate position on android-based mobile phone get very satisfactory result. Researchers (7) propose a plate detection algorithm based on saliency images applying a sliding window to identify the characters.

This study will detect the license plate number of vehicles using the CNN. The initial process is to create a training data 
license plate numbers using $\mathrm{CNN}$ processed on the server. Furthermore, the training data entered on the vehicle license plate detection applications. Vehicle license plate detection results will be displayed in percent accuracy.

\section{METHOD}

\section{A. Database Training}

The first step in this research is to prepare 546 vehicle image that will be used as database training. Then start the cropping process on the vehicle number plate using the labeling application. The crop image of the plate is stored in an .xml file. All crop files are collected and stored as .csv files. Although the size of cropping results is not the same, this is not a problem in the matching process. Unlike other methods that require the plate crop size to be exactly the same as the size of the plate being tested. The next process is segmentation and extrusion feature using CNN method with TensorFlow library and training result in the form of stored feature in the form of inference or extension .pb. This process is shown in Fig. 1.

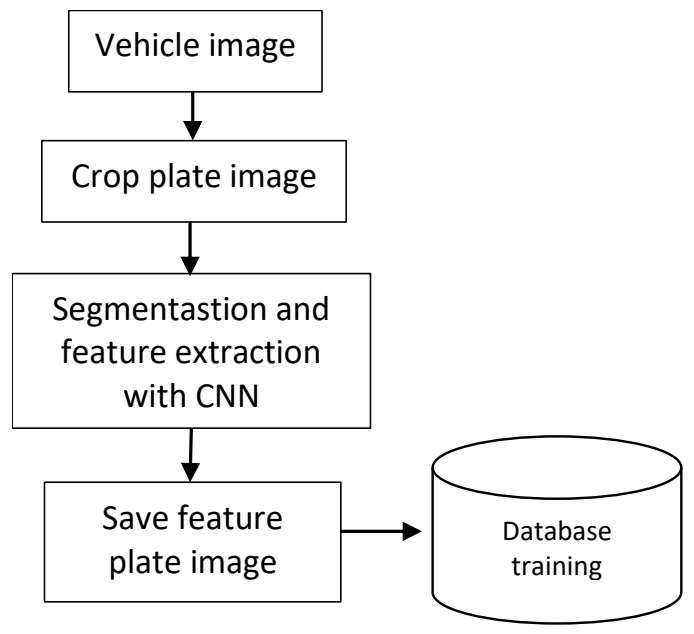

Fig. 1. Training process diagram.

\section{B. Convolutional Neural Network}

Convolutional Neural Network (CNN) is a very efficient pattern recognition algorithm that is widely used in image processing and pattern recognition (8). CNN has been well applied for the detection, segmentation, and recognition of objects and regions in images such as the introduction of traffic alerts, biological image segmentation of face detection, text, pedestrian and human body (9). CNN has many advantages such as simple structure, adaptability and few training parameters. CNN reduces the complexity of network models and the number of weights and has a unique advantage in image processing and speech recognition (8). CNN has four main operations (10), namely:

- Convolution Layer. The purpose of convolution on image data is to extract features from the input image. The convolution will produce a linear transformation of the input data according to the spatial information in the data. The weights on the layer specify the convolution kernel currently in use, so the convolution kernel can be trained based on the input on CNN.

- Rectified Linear Unit (ReLU). Aims to apply the sigmoid activation function to the activation output generated by the previous layer.

- Subsampling is the process of reducing the size of a data, according to (11) the use of a pooling layer on CNN only aims to reduce the size of the image so that it can be easily replaced with a convolution layer with the same stride as the corresponding pooling layer.

- Fully Connected Layer is a layer commonly used in the application of Multi Linear Perceptron and aims to transform the data dimension so that data can be classified in a linear fashion.

\section{License Plate Detection}

Initially, the detection process starts with getting vehicle input. Furthermore, the process of segmentation and feature extraction. The result of the process goes into the matching phase with the training database. The result of the process produces vehicle plate image. The system diagram is illustrated as shown in Fig. 2.

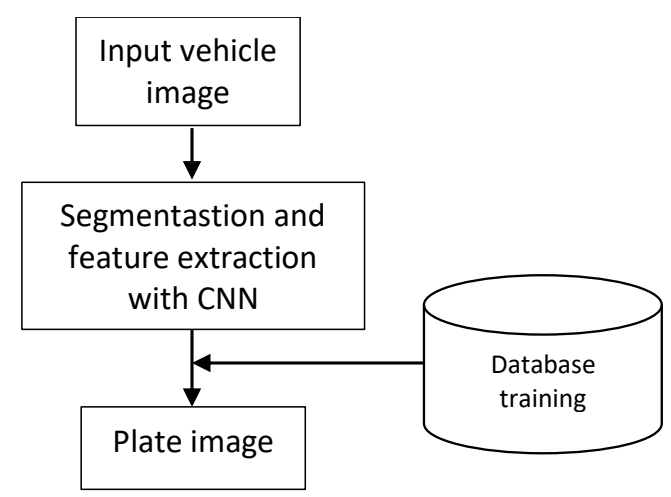

Fig. 2. License plate detection system diagram.

\section{IMPLEMENTATION}

This vehicle number plate detection application is implemented on Android-based mobile devices. Test data used in this research is to use vehicle image containing license plate of Indonesia country vehicle, vehicle image taken using camera of mobile device. There are 331 images of test data used. The test data consists of the image of motorcycles and cars. Fig. 3 shows an example of an application on a mobile phone.

Based on the testing, the results obtained were very satisfactory with the accuracy of detection of vehicle license numbers as much as $98.19 \%$, the remaining $1.81 \%$ of the plates were detected but not the image of the plate that was cropped. 


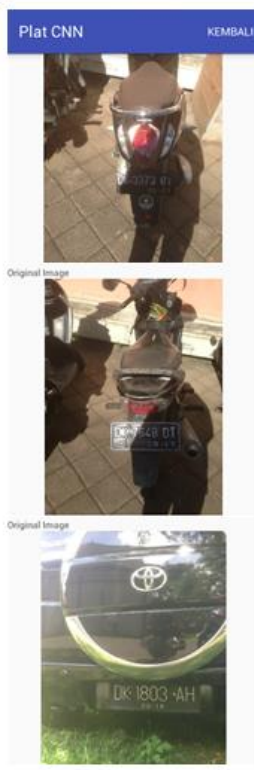

(a)

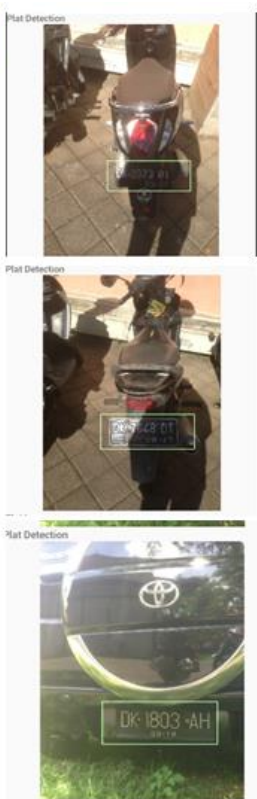

(b) (c)

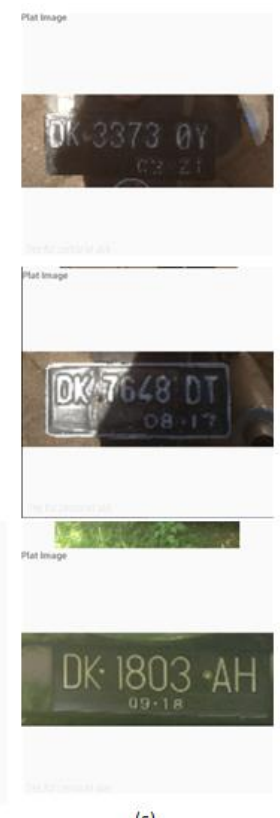

Fig. 3. Plate detection with non-uniform lighting (a) Vehicle images (b) detecting plat position (c) cropping.

It shows that the method has been implemented on CNN that training data and license plate detection is very pretty accurate in detecting vehicle license plate. There is an increase in results compared to previous studies conducted by sliding window method and histogram equalization (6). The results of vehicle plate detection shown in table 1. Fig. 4 shows an example of a missed detection on the vehicle.

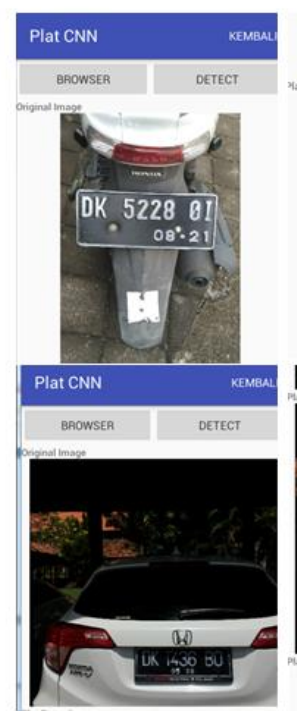

(a)
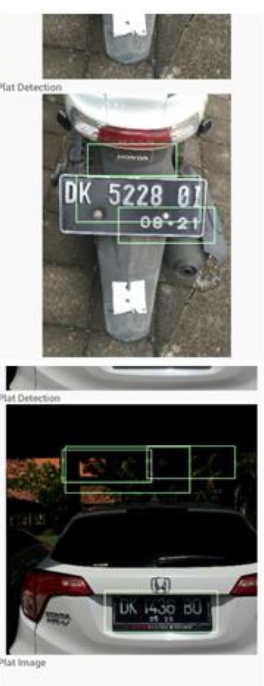

(b)
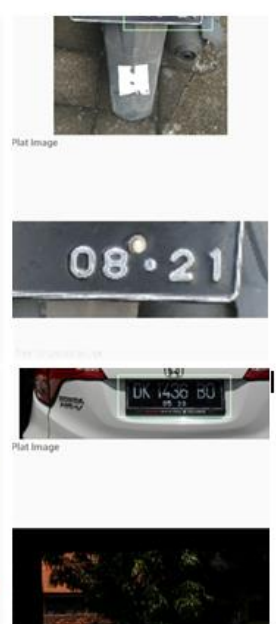

(c)

Fig. 4. Result Detection of non-vehicle plate (a) vehicle image (b) result of plate detection and negative image (c) Negative cropped images.

TABLE I. RESULT OF VEHICLE PLATE DETECTION

\begin{tabular}{|c|c|c|c|}
\hline $\begin{array}{c}\text { Code } \\
\text { Image }\end{array}$ & $\begin{array}{c}\text { Number of } \\
\text { Images }\end{array}$ & $\begin{array}{c}\text { Plate } \\
\text { Detected }\end{array}$ & $\begin{array}{c}\text { Not Detected } \\
\text { (Negative Image) }\end{array}$ \\
\hline P1 & 39 & 39 & 0 \\
\hline P2 & 49 & 47 & 2 \\
\hline P3 & 56 & 55 & 1 \\
\hline P4 & 49 & 48 & 1 \\
\hline P5 & 54 & 53 & 1 \\
\hline P6 & 84 & 83 & 6 \\
\hline & $\mathbf{3 3 1}$ & $\mathbf{3 2 5}$ & $\mathbf{6}$ \\
\hline
\end{tabular}

\section{CONCLUSION}

Implementation of CNN methods on android based mobile devices for data processing training and detection testing of vehicle license plate shows very satisfactory results. The accuracy of vehicle plate number detection is $98.19 \%$. Our research strongly recommends to use CNN method in the continuation of research of character recognition of vehicle license plate number.

\section{ACKNOWLEDGMENT}

The authors would like to thank of Directorate General for Research strengthening and Development, Ministry of Research, Technology and Higher Education, Republic of Indonesia as the sponsor of this research through the scheme of prime university research grants.

\section{REFERENCES}

[1] F.D. Kurpiel, R. Minetto, and B.T. Nassu, "Convolutional Neural Networks for License Plate Detection in Images," IEEE Proceed. [International Conference on Image Processing (ICIP); 2017 17-20 September, 2017].

[2] W. Zhou, H. Li, Y. Lu, and Q. Tian, "Principal Visual Word Discovery for Automatic License Plate Detection," IEEE Trans. on Image Process., vol. 21, no. 9, pp. 4269 - 4279, 2012.

[3] Y. Yuan, W. Zou, Y. Zhao, X. Wang, X. Hu, and N. Komodakis, "A Robust and Efficient Approach to License Plate Detection. IEEE Trans. on Image Process., vol. 26, no. 3, pp. 1102-1114, 2017.

[4] T.K. Cheang, Y.S. Chong, and Y.H. Tay, "Segmentation-free Vehicle License Plate Recognition using ConvNet-RNN," [International Workshop on Advanced Image Technology, 2017].

[5] J.L. Tan, S.A.R. Abu-Bakar, M. M. Mokji, "License Plate Localization Based on Edge-Geometrical Features Using Morphological Approach," IEEE Proceed. [International Conference on Image Processing, September, 2013]

[6] I. Astawa, I.G.N.B. Caturbawa, I.M. Sajayasa, and I.M.A.D.S. Atmaja, "Detection of License Plate Using Sliding Window, Histogram of Oriented Gradient, and Support Vector Machines Method," J. Phys. Conf. Series. vol. 953, no. 1, 2018.

[7] K.H. Lin, H. Tang, T. and S. Huang, "Robust License Plate Detection Using Image Saliency," IEEE Proceed. [International Conference on Image Processing, September. 2010].

[8] T. Liu , S. Fang, Y. Zhao, P. Wang, J. Zhang, Implementation of Training Convolutional Neural Networks. Cornell University Library: USA, 2015, pp. 1-10. 
[9] Y. Lecun, Y. Bengio, and G. Hinton, "Deep Learning," Nature, vol. 521, no. 7553, pp. 436-44, 2010.

[10] S. Das and J. Mukherjee, "Automatic License Plate Recognition Technique using Convolutional Neural Network," Int. J. Comp. App., vol. 69 , no. 4 , pp. 32-6, 2017
[11] J.T. Springenberg, A. Dosovitskiy, T. Brox, and M. Riedmiller, Striving for Simplicity: The All Convolutional Net, 2014. 